\title{
Über die Entwicklung des marinen parasitischen Phycomyceten Lagenisma coscinodisci (Lagenidiales)
}

\author{
E. SCHNEPF ${ }^{1} \&$ G. DREBES ${ }^{2}$ \\ ${ }^{1}$ Lebrstubl für Zellenlebre, Universität Heidelberg; Heidelberg \\ und \\ 2 Biologische Anstalt Helgoland (Litoralstation); \\ List/Sylt, Bundesrepublik Deutschland
}

\begin{abstract}
On the development of the marine, parasitic phycomycete Lagenisma coscinodisci (Lagenidiales). This paper concentrates on the study of vegetative development and sexual reproduction in Lagenisma coscinodisci Drebes. Before infecting a diatom cell (Coscinodiscus granii), the freshly released zoospores pass through two different cyst stages. The primary zoospores are kidney-shaped and laterally biflagellated. They form a primary cyst with a spiny cyst wall which is left by isomorphic secondary zoospores. The latter form a secondary cyst (sometimes perhaps repeatedly), which is smooth-walled. The secondary cyst germinates and infects a new diatom cell by means of an infecting tube which enters the cell through the gap between epi- and hypotheca and develops a new thallus. Sexual reproduction is induced by ageing of cultures. Two kinds of isomorphic, kidney-shaped, laterally biflagellated swarmers are produced as in zoosporogenesis. Female-determined swarmers settle down near the host cell and encyst with a more or less smooth wall (oogonia). Male-determined swarmers are obviously attracted by the oogonia and encyst close to them to form a more or less smooth-walled antheridium which drives a thin fertilization tube to the oogonium. During plasmogamy the oogonium develops a thick, short hypha. The cytoplasm with the two nuclei moves completely into this hypha, concentrates near the hyphal tip, and surrounds itself with a thick wall to become a resting spore (oospore) in which karyogamy takes place. The walls of cysts, tubes, hyphae and spores react positively with zinc-chlor-iodide. In the spines and walls of the primary cysts the network of 2 to $3 \mathrm{~nm}$ thick fibrils is more distinct than in the other walls.
\end{abstract}

\section{EINLEITUNG}

Lagenisma coscinodisci Drebes, 1968 (Lagenidiales), die von Sparrow (1973) zu den Sirolpidiaceen gestellt wird, parasitiert in centrischen Plankton-Diatomeen der Gattungen Coscinodiscus und Palmeria. Sie tritt in der Nordsee auf (Drebes, 1966, 1968, 1974), wurde aber auch an der Westküste der USA (Gotelli, 1971) und bei Jamaika (Grahame, 1976) gefunden. Die Morphologie des endobiotischen Thallus und seine Entwicklung ist bei Drebes $(1966,1968)$ beschrieben und mikrokinematographisch dokumentiert worden (Drebes, 1969). Der Infektionsverlauf ist von Grahame (1976) dargestellt. Chakravarty untersuchte die ökologischen Bedingungen der Infektion (1974) und die Möglichkeiten einer absoluten Reinkultur (1969). 
Wir beobachteten in Kulturen (zusammen mit dem Wirt, Coscinodiscus granii) bislang unbekannte Einzelheiten der vegetativen und die sexuelle Entwicklung des Pilzes. Dabei zeigte sich, daß der Sexualprozeß anders abläuft als bei den bisher untersuchten Vertretern der beiden anderen Familien der Lagenidiales, der Lagenidiaceae und der Olpidiopsidaceae (vgl. Sparrow, 1960, 1973). Bei den Sirolpidiaceae ist, nach Sparrow (1973), die Bildung echter Dauersporen und das Auftreten von Sexualprozessen noch nicht beobachtet worden.

\section{MATERIAL UND METHODEN}

Die für diese Untersuchungen verwendete Kultur stammt von einer am 18.9.1975 vorgenommenen Isolierung aus einer Planktonprobe vom Nordsylter Wattenmeer bei List ab. Um eine Klonkultur zu erhalten, wurde mit einem einzigen Pilzthallus gestartet.

Der Wirt, Coscinodiscus granii Gough, wurde in Petrischalen im bekannten $f / 2$ Medium (McLachlan, 1973) bei $15^{\circ} \mathrm{C}$ und 500-1000 Lux (Osram-Leuchtstoffröhren) in einem Licht-Dunkel-Rhythmus von 14 : 10 Stunden kultiviert. Die Kultur war nicht bakterienfrei. Der Parasit hat entsprechend den Kulturbedingungen und abhängig von der Wirtsgröße eine Generationsdauer von 1 bis 3 Tagen und muß wegen seines raschen Wachstums alle 4 bis 6 Tage übergeimptt werden.

Für die Lichtmikroskopie am lebenden Organismus verwendeten wir vorzugsweise die Tauchimmersionen der Fa. Leitz (25mal, 50mal, 100mal). Außerdem färbten wir Zellen mehrere Stunden mit Karminessigsäure, z. T. nach Vorfixierung mit Glutaraldehyd, hellten mit Phenol auf und untersuchten sie im Phasenkontrast.

Für die Elektronenmikroskopie ließen wir die Schwärmer sich auf befilmte Goldnetzchen festsetzen und weiterentwickeln. Die Präparate wurden dann, z. T. nach Fixierung mit Glutaraldehyd, nach Negativkontrastierung durch Phosphorwolframsäure oder Uranylacetat in einem Siemens Elmiskop 101 untersucht.

\section{ERGEBNISSE}

\section{Vegetativer Zyklus}

Eine Coscinodiscus-Zelle wird infiziert (vgl. Grahame, 1976), indem sich eine sekundäre Zoospore (s. u.) auf die Kieselschale setzt, eine glattwandige, kugelrunde Cyste bildet (Abb. 1, 4 L) und dann eine dünne (Durchmesser etwa 1,5-3,0 $\mu \mathrm{m}$ ) Infektionshyphe austreibt, die mit einer Geschwindigkeit von $0,5-1 \mu \mathrm{m}$ pro Minute mehr oder weniger gezielt auf die Gürtelfuge zwischen Epi- und Hypotheka zuwächst. Dabei bildet sich in der Cyste zunächst eine immer größer werdende Vacuole aus. Schließlich wandert das ganze Plasma in den Keimschlauch. Bevor sich dieser durch die enge Fuge quetscht, verbreitert er sich oft etwas. Wenn der Wirtsprotoplast erreicht ist, schwillt der Keimschlauch blasig an und entwickelt sich dann zum endobiotischen Thallus. Die auf der Außenseite der Diatomeen-Schale sitzende Cyste und der Keim- 

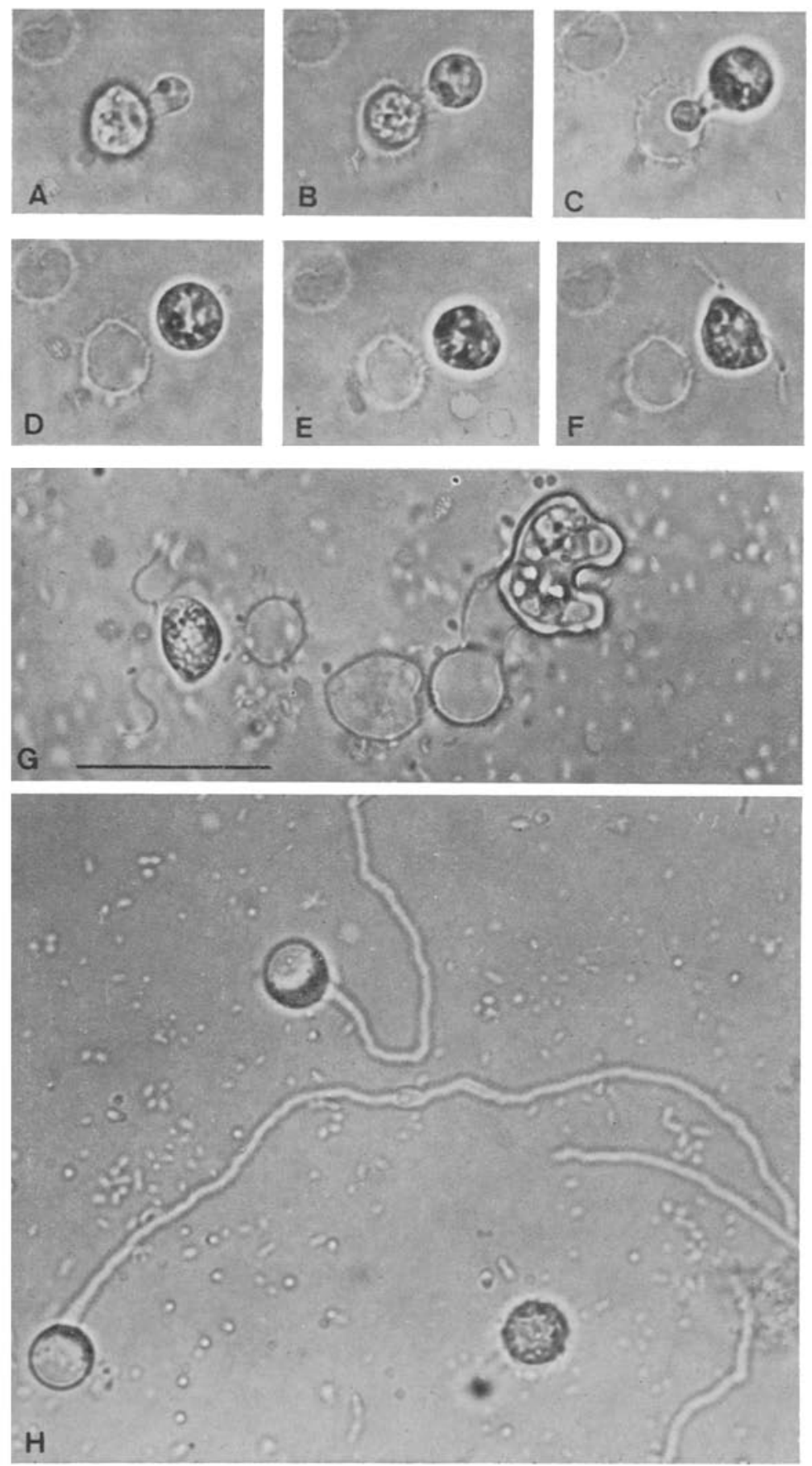

Abb. 1: $A-G$ Bildung einer sekundären Zoospore aus einer Primärcyste. A: $12^{49}, B: 12^{50}$, C: $12^{50,5}, \mathrm{D}: 12^{51}, \mathrm{E}: 12^{54}, \mathrm{~F}: 12^{55}$. G: Links ausgetretene Sekundär-Zoospore, rechts sexuelle Fortpflanzung. Größenvergleich von bestachelter Primärcyste und Gametangien. Unregelmäßige Dauerspore. $H$ Sekundärcysten mit lang ausgetriebenen Infektionsschläuchen und eine Primärcyste. (Marke: $20 \mu \mathrm{m}$ ) 
schlauch werden plasmafrei, bleiben aber erhalten. Wenn sich die Zoosporen auf dem Glas der Kulturschale und nicht auf einer Diatomee encystiert haben, kann der Infektionsschlauch bis über $80 \mu \mathrm{m}$ lang werden.

Die Entwicklung des endobiotischen Thallus ist bei Drebes (1966) beschrieben (vgl. auch Drebes, 1968, 1969, 1974; Grahame, 1976) und braucht deshalb hier nicht noch einmal dargestellt zu werden. In dem unseptierten, verzweigten Myzel entwickeln sich holocarp zahlreiche Zoosporen. Sie werden durch einen Entleerungstubus nach außen entlassen, der zwischen den beiden auseinanderweichenden Theken oder zwischen Gürtelbändern hindurch nach außen mündet und sich öffnet, wenn die Zoosporen beweglich geworden sind.

Die so entstandenen primären Zoosporen sind nierenförmig und haben zwei lateral inserierte Geißeln. Sie infizieren, entgegen den bisherigen Annahmen, nicht direkt eine neue Wirtszelle, sondern setzen sich nach kurzem Umherschwimmen fest und encystieren sich sehr schnell. Die rundlichen vegetativen Primärcysten (Abb. 1, $4 \mathrm{~L}$ und $5 \mathrm{~A}$ ) sind mit feinen Stacheln versehen. Thre Wand reagiert positiv mit Chlorzinkjod (Abb. 4) und ist deutlich aus einem Netzwerk feiner Fibrillen (Durchmesser etwa 2-3 nm) aufgebaut. Auch die Stacheln zeigen diese Fibrillen (Abb. 5 C).

Nach gewisser Zeit (20 Min. bis einige Stunden nach dem Freiwerden der primären Zoosporen) kriecht aus der Primärcyste durch eine kleine, trichterförmige Öffnung eine sekundäre Zoospore. Sie verläßt die Cyste (Abb. 1) mit dem Geißelpol voran; am entgegengesetzten Ende ist sie anfangs in einen Schwanz verlängert. Die Zoospore ändert aber schnell ihre Form und wird, wie die primäre Zoospore, nierenförmig. Die Geißeln bilden sich, während die Cyste verlassen wird, und wachsen innerhalb von 1 bis 2 Minuten zur normalen Größe heran. Diese sekundären Zoosporen können nun direkt einen neuen Wirt in der oben beschriebenen Weise befallen, indem eine meist etwas kleinere Sekundärcyste entsteht, die, wie erwähnt, stachellos ist. Man findet aber häufig auch leere, stachellose Cystenhüllen ohne Keimschlauch auf dem Boden der Kulturschale. Möglicherweise können die sekundären Zoosporen bei nicht zusagenden Bedingungen die sekundäre Cyste verlassen, ohne einen Keimschlauch zu bilden, und wieder frei herumschwimmen, um sich dann neu festzusetzen und sich zu encystieren. Die Wände der glatten Cysten und des Keimschlauches reagieren ebenfalls positiv mit Chlorzinkjod. Die Wandfibrillen sind jedoch weniger gut als bei den primären Cysten zu erkennen (Abb. 5 B). Dieser vegetative Zyklus läuft besonders in frisch angeimptten Kulturen $a b$, in denen die Zahl der Parasiten gering und die Zahl der Wirtszellen groß ist.

\section{Generativer Zyklus}

Wenn sich nach einigen Tagen der Parasit in den Kulturschalen stark vermehrt hat und nur noch wenige nichtinfizierte Coscinodiscus-Zellen vorhanden sind, triff man vermehrt auf Sexualstadien. Der Pilzklon bildet beide Geschlechter aus, aber ein Thallus produziert nach unseren bisherigen Beobachtungen nur männlich oder weiblich determinierte Schwärmer. Lagenisma coscinodisci hat also offenbar phänotypische Geschlechtsbestimmung. Die konträrgeschlechtlichen Thalli können aus derselben oder aus verschiedenen Wirtszellen stammen. 
Beide Typen von sexuell geprägten Thalli entlassen Schwärmer, die, jedenfalls soweit lichtmikroskopisch festzustellen ist, den Zoosporen ähneln, aber in der Regel etwas größer sind als diese (Abb. $1 \mathrm{G}$ ). Die weiblich determinierten Schwärmer setzen sich meist in unmittelbarer Nähe der Wirtszelle fest, encystieren sich und bilden damit Oogonien. Die männlich determinierten Schwärmer schwimmen, offenbar chemotaktisch geleitet, in die Nähe eines Oogoniums und encystieren sich dort ebenfalls.

Beide "Cysten", Oogonien und Antheridien, sind im wesentlichen glattwandig und tragen nur gelegentlich einzelne Stacheln. Sie unterscheiden sich morphologisch nicht. Sie sind meistens etwa gleich groß, das Oogonium ist gelegentlich etwas kleiner als das Antheridium, seltener ist es umgekehrt. Beide unterscheiden sich nicht von einer sekundären Cyste (Abb. 5 B), wenn man von der unterschiedlichen Größe absieht.

Knapp eine Stunde nach dem Freiwerden der Schwärmer bildet das Antheridium
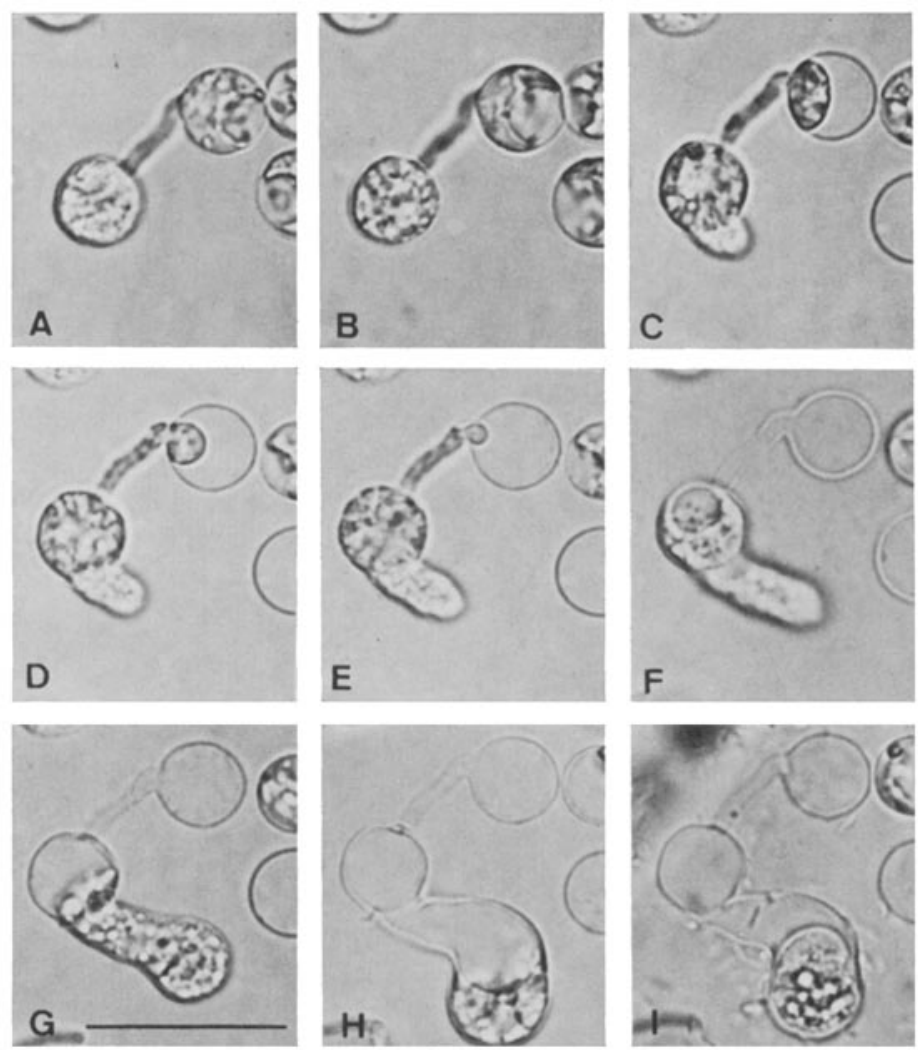

Abb. 2: $A-I$ Sexualprozeß und Dauersporenbildung. Serie von Photographien derselben $\mathrm{Ob}_{-}$ jektstelle. In $A\left(12^{15}\right)$ hat der Fusionsschlauch des Antheridiums das Oogonium erreicht, dabei entsteht im Antheridium eine Vacuole. In $B\left(12^{24}\right)$ tritt der Protoplast des Antheridiums in das Oogonium, das in $C\left(12^{50}\right)$ eine kurze, dicke Hyphe bildet. In $D\left(13^{05}\right)$ und $E\left(13^{13}\right)$ entleert sich das Antheridium weiter, die Hyphe verlängert sich. In $F\left(13^{25}\right)$ beginnt die Auswanderung des Plasmas aus dem Oogonium unter Bildung einer Vacuole. In $G\left(14^{19}\right)$ ist das Oogonium fast entleert. In $H\left(18^{45}\right)$ hat sich das Protoplasma in der Hyphenspitze zusammengeballt und in $I$ ( $11^{20}$ des übernächsten Tages) mit einer dicken Wand umgeben. (Marke: $20 \mu \mathrm{m}$ ) 
einen Befruchtungsschlauch (Abb. 2, 3, 4) (Dicke etwa 1,5-3,0 $\mu \mathrm{m}$ ), der, offenbar wiederum chemotaktisch angelockt, auf das Oogonium zuwächst und die Cystenwand durchbricht, wobei es im Oogon oft zu einer Art Abwehrreaktion kommt, indem wie bei einer Infektion Wandmaterial an dieser Stelle abgelagert wird (Abb. $4 \mathrm{D}$ und $\mathrm{H}$ ).
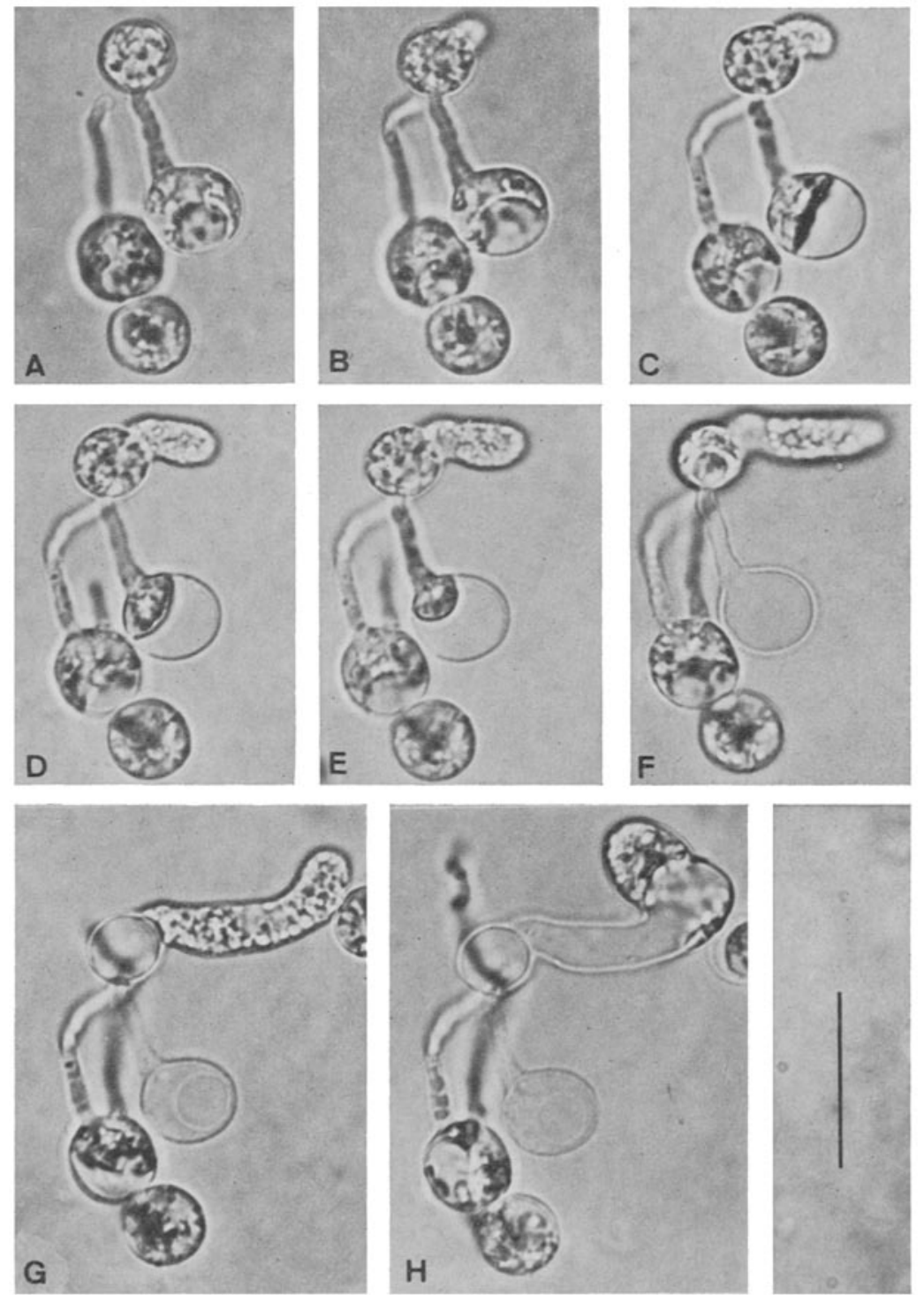

Abb. 3: $A-H$ Sexualprozeß, Ausschaltung einer Mehrfachbefruchtung. 3 Antheridien treiben je einen Befruchtungsschlauch auf ein Oogonium; nur dem Erstankömmling gelingt die Fusion. Das 2. Antheridium, das fast gleichzeitig das Oogonium erreicht hat, stellt das Wachstum ein, der Befruchtungsschlauch des 3. Antheridiums wächst an der Zygote vorbei. $A 12^{15}, B 12^{24}$, $C 12^{50}, D 13^{05}, E 13^{15}, F 13^{55}, G 14^{19}, H 18^{45}$. (Marke: $20 \mu \mathrm{m}$ ) 

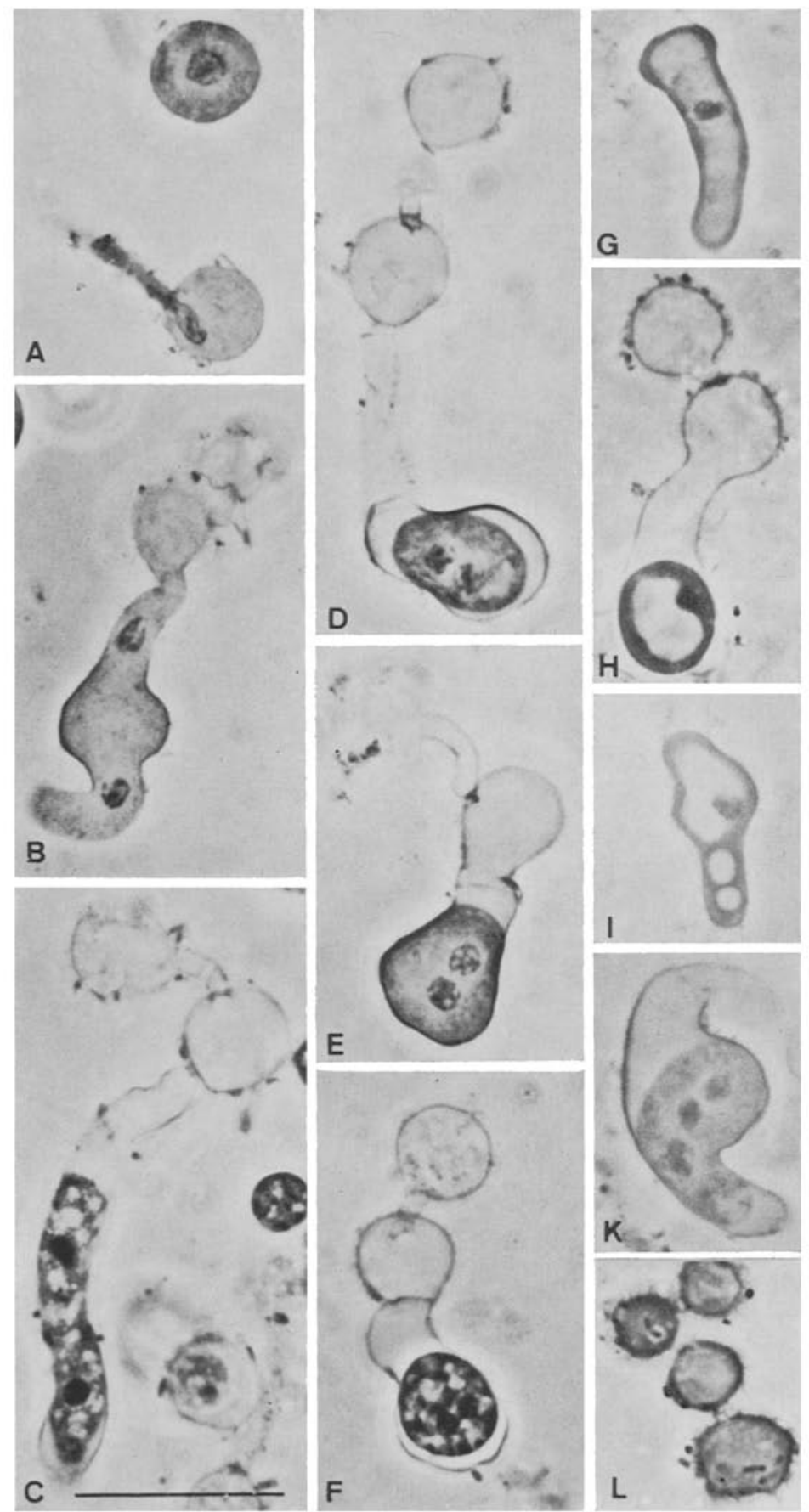

Abb. 4: $A-K$ Karminessigsäurefärbung, $C$ und $F$ nach Glutaraldehydfixierung; $L$ Chlorzinkjodfärbung. $A$ Antheridien, das untere keimt aus, wobei der Kern gerade in den Fusionsschlauch wandert. $B-F \mathrm{Z}$ wei Kerne in der Hyphe, die aus dem Oogon nach der Plasmogamie auswächst, und in jungen Dauersporen. $G$ Karyogamie in einer unreifen Dauerspore. $H-I$ Fast fertige Dauersporen nach der Karyogamie mit einem bzw. drei großen Lipidtropfen. $K \mathrm{Ab}$ normer dreikerniger Plasmaballen kurz vor der Dauersporenbildung. $L$ Leere (vegetative) Primärcysten mit feinen Stacheln; hier wie z. T. auch in den anderen Teilabbildungen sind die Wände von Bakterien besetzt. (Marke: $20 \mu \mathrm{m}$ ) 

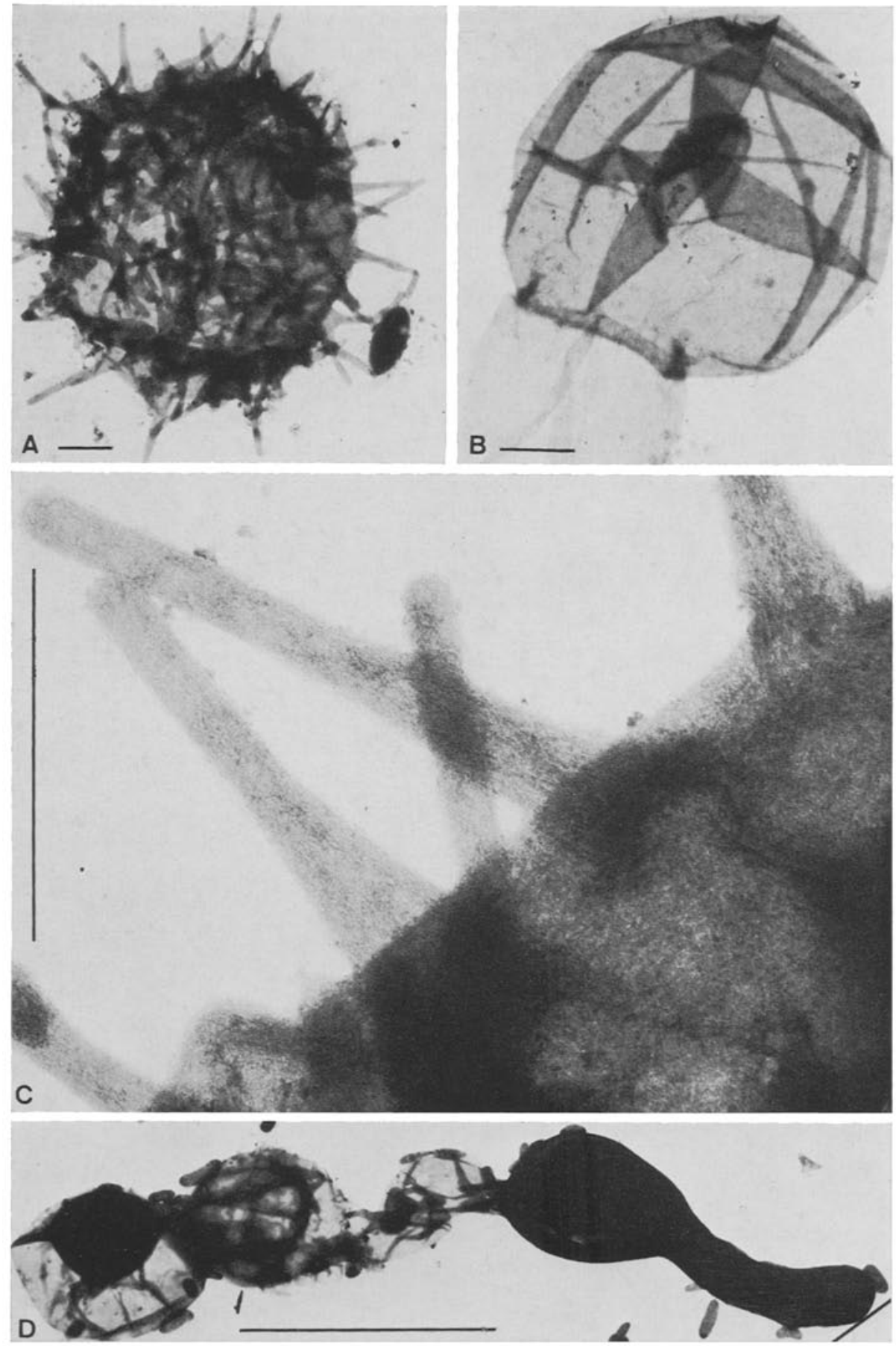

Abb. 5: $A-D$ Elektronenmikroskopisch nach schwacher Negativ-Kontrastierung mit Phosphorwolframsäure $(A, C, D)$ oder Uranylacetat $(B) . A$ Leere, vegetative Primärcysten (Marke: $1 \mu \mathrm{m}) . B$ Ausgekeimte, entleerte Sekundärcyste oder entleertes Antheridium (Marke: $1 \mu \mathrm{m}$ ). $C$ Ausschnitt aus $A$, Fibrillen in der Cystenwand und in den Stacheln (Marke: $1 \mu \mathrm{m}) . D$ Antheridium (links), Oogonium und junge Dauerspore (rechts) (Marke: $10 \mu \mathrm{m}$ ) 
Die Spitze des Befruchtungsschlauches öffnet sich. Das Plasma des Antheridiums wandert aus der Cyste, wobei sich wiederum an dem Pol, der dem Befruchtungsschlauch entgegengesetzt ist, eine Vacuole bildet. Der Zellkern, der vorher rund war, nimmt beim. Ubertritt in den Befruchtungsschlauch Spindelform an (Abb. 4 A).

Auch wenn kein Oogon in unmittelbarer Nähe ist, wachsen die Antheridien zu einem dann oft gekrümmten, gelegentlich sogar verzweigten Keimschlauch aus. In Ausnahmefällen entstehen auch 2 Hyphen an einem Antheridium.

Wie man an Oogonien sehen kann, die das Ziel mehrerer Befruchtungsschläuche sind, hört die Anlockung des Oogons auf, wenn die Gametangiogamie begonnen hat. Die zu spät kommenden Befruchtungsschläuche stellen dann ihr Wachstum nach einiger Zeit ein oder wachsen am Oogon vorbei (Abb. 3).

Kurz nach Einleitung der Gametangiogamie bildet das Oogonium eine meist etwa 4 bis $10 \mu \mathrm{m}$ breite, of unregelmäßig geformte Hyphe, die bis etwa $50 \mu \mathrm{m}$, meist etwa $20 \mu \mathrm{m}$ lang wird. In diese Hyphe wandert das gesamte Plasma mit den beiden Zellkernen (Abb. $4 \mathrm{~B}$ ); das Oogonium und der angrenzende Teil der Hyphe entleert sich (Abb. $4 \mathrm{C}$ ), das Plasma klumpt sich, meist nahe oder in der Hyphenspitze, zusammen und umgibt sich innerhalb der Hyphe mit einer allmählich dicker werdenden Zellwand. Dieser Prozeß beginnt etwa 3 Stunden nach dem Freiwerden der Schwärmer. Der ganze Vorgang ist in seiner zeitlichen Abfolge in Abbildung 2 dargestellt. Abbildung $5 \mathrm{D}$ zeigt ein Oogonium mit Antheridium und Zygotenhyphe im elektronenmikroskopischen Bild.

In der sich zur Dauerspore (Oospore) entwickelnden Zygote wandern die beiden Kerne aufeinander zu und fusionieren schließlich (Abb. $4 \mathrm{G}$ ). Dann fließen die vielen kleinen Lipoidtröpfchen zu einem (Abb. $4 \mathrm{H}$ ) oder wenigen (Abb. $4 \mathrm{I}$ ) großen Tropfen zusammen. Die Verdickung der Wand der Dauerspore ist nach 1 bis 2 Tagen abgeschlossen. Meist haben die Dauersporen eine unregelmäßige Form (Abb. 1 G). Die Hyphenwand, die sie umhillt, wird manchmal faltig.

Die Dauersporen machen anscheinend nun eine längere Ruheperiode durch. Es ist uns jedenfalls bislang noch nicht gelungen, sie wieder zum Auskeimen zu bringen.

Die beschriebenen Prozesse können auch im Inneren einer Coscinodiscus-Zelle ablaufen, wenn sie von mehreren Thalli infiziert war und die Schwärmer nicht alle nach außen kamen.

Die Wände der Gametangien, des Fusionsschlauches, der Zygotenhyphe und der Dauerspore reagieren ebenfalls positiv mit Chlorzinkjod, die Fibrillen sind jedoch weniger gut als bei den Primärcysten erkennbar.

Es ist nicht auszuschließen, daß unter anderen Bedingungen oder in Sonderfällen die Entwicklung im Detail anders verlaufen kann. So beobachteten wir gelegentlich abnorm große und dann mehrkernige Zoosporen und sexuell geprägte Schwärmer und, wohl als Folge davon, junge Dauersporen mit mehr als zwei Kernen (Abb. $4 \mathrm{~K}$ ).

\section{DISKUSSION}

Unsere Befunde über die generative und vegetative Entwicklung von Lagenisma sind von Bedeutung für die taxonomische Einordnung dieses Pilzes, der im Haushalt 
des Meeres durchaus eine Bedeutung hat. Sie zeigen außerdem, daß dieser Pilz ein geeignetes Objekt für zellbiologische und entwicklungsphysiologische Fragestellungen ist. Ungeklärt ist z. B., wie die männlich determinierten Schwärmer und die Befruchtungsschläuche durch die Oogonien angelockt werden und was den Umschlag von der vegetativen zur sexuellen Fortpflanzung steuert; vielleicht spielt bei dieser Umstimmung eine Anhäufung von Stoffwechselprodukten des Parasiten eine Rolle.

Eine Folge von mehreren Generationen von Zoosporen ist von vielen anderen Oomyceten bekannt. Bei Lagenisma sind die fertigen Schwärmer aller Typen nierenförmig und lateral begeißelt. Es handelt sich hierbei also nicht um eine Diplanie im engen Sinne (mit morphologisch verschiedenen Zoosporen; Gäumann, 1964). Allerdings haben wir es mit einer "Dicystie" zu tun, denn die primäre vegetative Cyste ist bestachelt, die sekundäre (wie die generativen Cysten, $\mathrm{d}, \mathrm{h}$. Antheridien und Oogonien) glatt. Durch die Bildung von Gametangien direkt aus Schwärmern, die den Zoosporen morphologisch gleichen, verläuft der Sexualzyklus völlig anders als bei den Lagenidiaceae und Olpidiopsidaceae (vgl. Sparrow, 1960, 1973). Da bei den übrigen Vertretern der Sirolpidiaceae die generative Vermehrung unbekannt ist oder fehlt, ist es nicht möglich, zu entscheiden, ob die Zuordnung von Lagenisma in diese Familie (Sparrow, 1973) durch unsere Befunde gestürzt ist.

Über den Ort, den Zeitpunkt und den Ablauf der Meiose läßt sich noch nichts sagen. Zur Klärung der Frage sollte besonders Augenmerk auf das Auskeimen der Dauersporen (Oosporen) gerichtet werden.

Die positive Reaktion der Wände mit Chlorzinkjod und ihre Fibrillenstruktur deuten darauf hin, daß sie aus Zellulose bestehen. Eine genaue Identifizierung erlauben aber diese Methoden nicht.

Danksagungen. Wir danken Frl, G. Deichgräber für ihre umsichtige Mitarbeit, Frl. D. Sill (List) für die Unterhaltung der Kulturen und Frau H. Harbst (List) für ihre Hilfe im Photolabor. Die Deutsche Forschungsgemeinschaft unterstützte die Arbeit mit Sachbeihilfen.

\section{ZITIERTE LITERATUR}

Chakravarty, D. K., 1969. Zum Kulturverhalten des marinen parasitischen Pilzes Lagenisma coscinodisci. Veröff. Inst. Meeresforsch. Bremerh. 11, 309-312.

- 1974. On the ecology of the infection of the marine diatom Coscinodiscus granii by Lagenisma coscinodisci in the Weser estuary. Veröff. Inst. Meeresforsch. Bremerh. (Suppl.) 5, 115-122.

Drebes, G., 1966. Ein parasitischer Phycomycet (Lagenidiales) in Coscinodiscus. Helgoländer wiss. Meeresunters. 13, 426-435.

- 1968. Lagenisma coscinodisci gen. nov. spec. nov., ein Vertreter der Lagenidiales in der marinen Diatomee Coscinodiscus. Veröff. Inst. Meeresforsch. Bremerh. Sonderbd. 3, 67-70.

- 1969. Lagenisma coscinodisci (Lagenidiales) - Vegetative Vermehrung in der Kieselalge Coscinodiscus granii. Begleitveröff. zum Film E 1398. Enc. Cin., Göttingen, 1-8.

- 1974. Marines Phytoplankton. Thieme, Stuttgart, 186 pp.

Gäumann, E., 1964. Die Pilze. 2. Aufl. Birkhäuser, Basel, 541 pp.

Gotelli, D., 1971. Lagenisma coscinodisci, a parasite of the marine diatom Coscinodiscus occurring in the Puget Sound, Washington. Mycologia 63, 171-174.

Grahame, S., 1976. The occurrence of Lagenisma coscinodisci in Palmeria bardmania from Kingston harbour, Jamaica. Br. phycol. J. 11, 57-61. 
McLachlan, J., 1973. Growth media - marine. In: Handbook of phycological methods, culture methods and grow th measurements. Ed. by J. R. Stein. Cambridge University Press, Cambridge, $25-51$.

Sparrow, F. K. Jr., 1960. Aquatic phycomycetes. Univ. Michigan Press, Ann Arbor, 1187 pp.

- 1973. Lagenidiales. In: The fungi. Ed. by G. C. Ainsworth, F. K. Sparrow \& A. S. Sussman. Acad. Press, New York, 4 B, 159-163.

Anschrift des erstgenannten Autors: Prof. Dr. E. Schnepf

Lehrstuhl für Zellenlehre

Universität Heidelberg

Im Neuenheimer Feld 230

D-6900 Heidelberg

BRD 